Carnets de géographes

GÉOGRAPHES.

\title{
Le petit prince ou comment faire lieu ? Entretien imaginaire avec Antoine de Saint Exupéry
}

\section{Philippe Gervais-Lambony}

\section{(2) OpenEdition}

\section{Journals}

Édition électronique

URL : http://journals.openedition.org/cdg/293

DOI : $10.4000 /$ cdg. 293

ISSN : 2107-7266

Éditeur

UMR 245 - CESSMA

Référence électronique

Philippe Gervais-Lambony, « Le petit prince ou comment faire lieu ? Entretien imaginaire avec Antoine de Saint Exupéry », Carnets de géographes [En ligne], 8 | 2015, mis en ligne le 01 septembre 2015, consulté le 30 avril 2019. URL : http://journals.openedition.org/cdg/293 ; DOI : 10.4000/cdg.293

Ce document a été généré automatiquement le 30 avril 2019

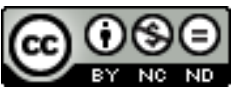

La revue Carnets de géographes est mise à disposition selon les termes de la Licence Creative Commons Attribution - Pas d'Utilisation Commerciale - Pas de Modification 4.0 International. 


\title{
Le petit prince ou comment faire lieu ? Entretien imaginaire avec Antoine de Saint Exupéry
}

\author{
Philippe Gervais-Lambony
}

\begin{abstract}
Aller vite ? Mais aller où ? (...) « Paris-Marseille en un quart d'heure, c'est formidable ! » Car vos fils et

vos filles peuvent crever : le grand problème à résoudre sera toujours de transporter vos viandes à la vitesse de l'éclair. Que fuyez-vous donc ainsi, imbéciles? Hélas! C'est vous que vous fuyez, vous même - chacun de vous se fuit soi-même, comme s'il espérait courir assez vite pour sortir enfin de sa gaine de peau... On ne comprend rien à la civilisation moderne si l'on n'admet pas d'abord qu'elle est une conspiration universelle contre toute espèce de vie intérieure.
\end{abstract}

Georges Bernanos, La France contre les robots, 1946, p. 82. Le texte que l'on va lire est le résultat d'un travail de recherche sur l'œuvre d'Antoine de Saint-Exupéry. Proposer de mobiliser la littérature comme objet de recherche pour une approche géographique est une démarche partagée par beaucoup d'auteurs, les études de ce type sont nombreuses et dépassent le champ d'une spécialité qui pourrait être qualifiée de "géographie littéraire ». Les manières de conduire une telle démarche sont diverses, Michel Collot (2014) a proposé une catégorisation de ces approches, depuis l'analyse des référents spatiaux des œuvres littéraires (qui peut conduire à comprendre comment la littérature transfigure les lieux ou nous les rend compréhensibles) jusqu'à ce qu'il définit comme la "géo-poétique », à savoir l'étude de l'espace de l'œuvre elle-même, produite par l'écriture, en passant par la «géo-critique » qui vise à analyser l'espace 
décrit par l'œuvre indépendamment des référents spatiaux. On peut aussi distinguer les travaux qui mobilisent un ensemble de textes d'auteurs différents ayant pour référent le même lieu, et ceux qui, au contraire, partent d'une œuvre littéraire unique ou de l'ensemble de la production d'un auteur. C'est de cette seconde catégorie que ressort le présent texte. Il est intitulé « entretien » car ce n'est pas tant d'une étude des référents spatiaux ou de la spatialité du texte qu'il est question, que d'une rencontre (faite, comme souvent, par hasard) avec un auteur (mais aussi avec ses personnages) et d'une analyse de la relation humaine au monde qui parcoure son œuvre. Entretien imaginaire bien sûr, et forcément influencé par celui qui le conduit et qui dans sa rencontre personnelle avec les textes et la pensée d'un auteur oriente ses questions vers ses propres préoccupations et celles du moment; entretien qui s'appuie principalement sur Le Petit Prince mais mobilise aussi d'autres ouvrages de Saint-Exupéry. Les porosités entre la géographie et l'œuvre de Saint-Exupéry sont, on le verra, nombreuses, on se sentira donc libre ici de considérer cette œuvre comme à la fois littéraire et géographique puisqu'elle est bel et bien la restitution d'une pensée sur l'espace et sur la relation humaine à l'espace. Un titre comme Terre des hommes le dit assez puisqu'il pose en fait la question : comment les hommes fontils de la terre (leur « planète »), leur lieu?

2 La littérature de la première moitié du XXème siècle est d'ailleurs profondément habitée par cette question et l'on comprend pourquoi, marquée qu'elle fut par deux conflits mondiaux. Comment, malgré tout, "être humains sur la terre» (Berque, 1996) ? La littérature des années 1940 est tout particulièrement imprégnée de cette question et l'inquiétude face à ce qui est vécu comme une sorte de basculement et effondrement du monde, que bien des auteurs assimilent à la « modernité ». Et au cœur de la définition de cette dernière est l'idée d'accélération. Récurrence d'images d'un monde qui va de plus en plus vite et perd le sens de la durée. Georges Bernanos, en exil au Brésil au moment où Saint-Exupéry l'est aux Etats-Unis, porte, violemment, cette même accusation contre la modernité dans la citation placée en exergue du présent texte.

Dans ce sentiment de brusque compression de l'espace et du temps, ou plus exactement de l'espace par le temps (Harvey, 1990), la question de la possibilité de la lenteur, voire de l'immobilité, devient centrale mais en cache une autre qui est directement spatiale: l'accélération interdit-elle de «faire lieu »? On doit entendre ici «lieu» comme ce qui définit un " habiter » et permet aux humains d'être au monde (Paquot, 2005 ; Lazzaroti, 2006). Cette interrogation, qui porte sur les relations entre espace et temps, traverse les sciences humaines. La géographie comme les autres, mais tout de même centralement puisque la dimension spatiale des sociétés est son objet majeur, puisqu'elle cherche à dire comment l'espace est fait lieu par les êtres humains qu'y l'habitent. A ce point, la géographie ne me semble pas pouvoir se passer de la littérature, et peut-être arrive-t-il que l'inverse soit aussi vrai?

\section{Le Petit Prince, une manière de géographie}

Le Petit Prince se caractérise, dans son fond comme dans sa forme, par l'absence de nécessité. Le Petit Prince est un livre qui semble n'être, en effet, pas à sa place ni dans le temps ni dans l'espace. Il a été écrit pendant la seconde guerre mondiale, à un moment où l'important aurait dû sembler ailleurs et certainement l'était. Le moment est dramatique pour le monde mais aussi dans la vie de Saint Exupéry ${ }^{1}$ : il a fuit la France de Vichy (sans pour autant vouloir rejoindre De Gaulle à Londres), perdu ses amis les plus chers, ses 
compagnons de l'aéropostale, Mermoz et Guillaumet, morts en vol respectivement en 1936 et en 1940, plus largement, et depuis le succès de Vol de nuit, la petite société des pilotes professionnels regarde Saint-Exupéry avec méfiance voire hostilité (beaucoup considèrent qu'il a trahi cette confrérie en faisant de leurs expériences matière à littérature pour sa gloire personnelle). Saint-Exupéry est donc seul et désespère du monde et des hommes, c'est à cette époque de sa vie qu'il dit haïr son époque, ce dont lui fera reproche Albert Camus quelques années plus tard:

si bouleversant que ce soit, ce cri, venant de lui qui a tant aimé les hommes dans ce qu'ils ont d'admirable, nous ne le prendrons pas à notre compte. Quelle tentation, pourtant, à certains heures, de se détourner de ce monde morne et décharné ! Mais cette époque est la nôtre et nous ne pouvons vivre en nous haïssant (1953 : 140).

En réalité, Saint-Exupéry certes doute de tout mais, comme Camus, il ne supporte surtout pas d'être exclu de l'action, d'être laissé de côté. Il s'efforce d'écrire ce qu'il pense devoir être sa grande œuvre, livre qu'il n'achèvera pas (Citadelle ne sera publié que quatre ans après sa mort) tout en cherchant par tous les moyens à se faire remobiliser dans l'aviation militaire. C'est le moment où son éditeur américain lui propose d'écrire un livre pour enfants. Une particularité de Saint-Exupéry est le caractère immédiatement international de son œuvre. Il se rend plusieurs fois à New York dès les années 1930, Vol de nuit est un succès immédiat aux Etats-Unis. Terre des Hommes est traduit avant même d'être publié en France. Pilote de guerre (Flight to Arras) est écrit à destination du public états-unien pour convaincre de la nécessité de l'entrée en guerre des Etats-Unis. Le Petit Prince n'échappe pas à cette règle : il est publié simultanément en anglais et en français.

6 Saint-Exupéry lorsqu'il écrit Le Petit Prince juge ne pas être à sa place à New York, c'est en Afrique du Nord qu'il devrait être. Et les personnages principaux du livre ne sont pas non plus à leur place : le Petit Prince erre loin de chez lui, l'aviateur ne doit d'être là qu'à une panne de son avion. La structure même du livre semble refléter cet état de "déplacement», il se présente comme une simple juxtaposition: 27 chapitres, de longueurs très inégales, accompagnés de dessins de l'auteur, dont l'ordre pourrait être modifié sans conséquences majeures, dont on pourrait aussi supprimer certains sans grand préjudice pour l'ensemble. Un texte inutile donc, qui est même un effort pour cela, se distraire de l'important et d'un présent très sombre. C'est peut-être cette inutilité même qui, cependant, explique l'extraordinaire succès de ce conte philosophique qui se présente comme un livre pour enfants.

7 Le Petit Prince est en effet une œuvre mondialement connue, traduit en plus de 250 langues c'est un des livres les plus vendus au monde. Il raconte l'histoire d'un aviateur tombé en panne dans le Sahara et qui, dans ce désert, fait la rencontre d'un jeune garçon, un enfant, mystérieux. Le lecteur, en même temps que l'aviateur, va peu à peu découvrir qui est cet enfant, d'où il vient et ce qu'il cherche. Il a quitté son astéroïde, a voyagé de planète en planète ( 7 au total) et cherche à rentrer chez lui. Au cours du livre il raconte à l'aviateur ses expériences, ses découvertes, comme un explorateur. L'aviateur, qui est aussi le narrateur, joue ici le rôle du géographe qui habite la sixième planète (chapitre $\mathrm{XV}$ ) visitée par le Petit Prince : "le géographe est trop important pour flâner. Il ne quitte pas son bureau. Mais il y reçoit des explorateurs. Il les interroge, et il prend en note leurs souvenirs " ${ }^{2}$. Le géographe, ce "vieux monsieur qui écrivait d'énormes livres", explique ainsi sa complémentarité avec les explorateurs. Est-ce bien une question de complémentarité ou plutôt d'une double face du géographe? Double ou triple d'ailleurs, car on peut aussi bien 
reconnaître Saint-Exupéry dans l'aviateur (qu'il fut) que dans le Petit Prince lui-même (en quête de son passé et de ses lieux) que dans le géographe (qui écrit des livres).

Le Petit Prince peut donc à plusieurs titres être considéré comme un livre de géographie écrit par un géographe. D'où le désir d'écrire le présent texte sur un livre dont l'objet est bien d'expliquer ce qui fait lieu pour les humains, ce qu'être au monde pourrait vouloir dire. Ceci à travers une expérience vécue individuelle, celle de Saint-Exupéry lui même dont on trouve dans Le Petit Prince condensées tous les thèmes majeurs. On verra plus loin comment Saint-Exupéry est géographe au sens où il interroge et met en cause constamment les concepts de la discipline (distance, étendue, échelle, éloignement...). Ses interrogations, l'aviateur Saint-Exupéry les rencontre dans son expérience, il faut lire le premier chapitre de Terre des Hommes pour s'en convaincre. On y verra le pilote Guillaumet faire une leçon de géographie de l'Espagne au jeune Saint-Exupéry qui s'apprête à réaliser son premier vol Toulouse-Dakar :

mais quelle étrange leçon de géographie je reçus là ! Guillaumet ne m'enseignait pas l'Espagne ; il me faisait de l'Espagne une amie. Il ne me parlait ni d'hydrographie, ni de populations, ni de cheptel. Il ne me parlait pas de Guadix, mais des trois orangers qui, près de Guadix, bordent un champ : - Méfie-toi d'eux, marque les sur ta carte. Et les trois orangers y tenaient désormais plus de place que la Sierra Nevada (...) Et, peu à peu, l'Espagne de ma carte devenait, sous la lampe, un pays de conte de fées. Je balisais d'une croix les refuges et les pièges. Je balisais ce fermier, ces trente moutons, ce ruisseau. Je portais, à sa place exacte, cette bergère qu'avaient négligée les géographes. (Terre des Hommes, p. 176).

\section{Le lieu perdu des " grandes personnes »}

Le Petit Prince est un conte pour enfant, mais l'auteur nous glisse au détour d'une page qu'il faut néanmoins le prendre très au sérieux : «je n'aime pas qu'on lise mon livre à la légère ». L'enfance c'est du sérieux, c'est même la seule chose sérieuse et les enfants, tout comme le Petit Prince, savent bien ce qui est important et ce qui ne l'est pas. C'est précisément pourquoi le livre est dédié aux enfants mais aussi aux enfants devenus adultes :

je veux bien dédier ce livre à l'enfant qu'a été autrefois cette grande personne. Toutes les grandes personnes ont d'abord été des enfants. (Mais peu d'entre elles s'en souviennent). Je corrige donc ma dédicace: A Léon Werth quand il était un petit garçon ${ }^{3}$.

Dans un temps de doute et d'exil Saint-Exupéry se tourne ainsi vers ses valeurs les plus fortes, l'amitié et l'enfance, et produit l'œuvre qui lui donnera la plus grande postérité et qui est comme un condensé de son expérience humaine. Le Petit Prince sera donc une réflexion sur l'enfance, mais l'enfance chez Saint-Exupéry comme chez Proust est à la fois un temps et un lieu, on ne peut le dire plus clairement : «l'enfance, ce grand territoire d'où chacun est sorti ! D'où suis-je ? Je suis de mon enfance. Je suis de mon enfance comme d'un pays » ( Pilote de guerre: 158). Le Petit Prince traite d'un moment particulier de cette enfance, celui où on la quitte, et c'est donc tout autant quitter un temps que quitter un lieu. Ce moment est essentiel car il dévoile qu'espace et temps ne sont pas dissociables dans le vécu individuel en ce sens que les lieux n'existent que dans un temps donné et disparaissent donc en même temps que les liens humains qui y avaient été tissés et les avaient produits en les rendant uniques. Pas de retour possible donc sur un lieu même si l'on peut revenir au même "endroit », c'est-dire que l'espace, tout comme le temps, est caractérisé par l'irréversibilité... à moins que par le rêve : 
(...) quand me saisit le désir de revoir le côté de Guermantes, on ne le satisferait pas en me menant au bord d'une rivière où il y aurait d'aussi beaux nymphées que dans la Vivonne, pas plus que le soir en rentrant (...) je n'aurais souhaité que vînt me dire bonsoir une mère plus belle et plus intelligente que la mienne. Non ; (...) ce que je veux revoir, c'est le côté de Guermantes que j'ai connu (...) ; ce sont ces prairies où, quand le soleil les rend réfléchissantes comme une mare, se dessinent les feuilles des pommiers, c'est ce paysage dont parfois, la nuit dans mes rêves, l'individualité m'étreint avec une puissance presque fantastique et que je ne peux plus retrouver au réveil. (Marcel Proust, Du côté de chez Swann).

Mais Saint-Exupéry est plus pessimiste que Proust :

(...) je me souviens des jeux de mon enfance, du parc sombre et doré que nous avions peuplé de dieux, du royaume sans limite que nous tirions de ce kilomètre carré jamais entièrement connu, jamais entièrement fouillé. Nous formions une civilisation close, où les pas avaient un goût, où les choses avaient un sens qui n'était permis dans aucune autre. (...) Dans cet infini on ne rentrera jamais plus, car c'est dans le jeu, et non dans le parc, qu'il faudrait rentrer (Terre des hommes, p. 258).

11 Les «grandes personnes» du Petit Prince ne sont en tous cas pas capables de ce voyage immobile dans le temps et dans l'espace qui leur permettrait de vivre à nouveau les liens qui firent leurs lieux. C'est une conclusion essentielle du Petit Prince : méditation sur l'enfance, il est aussi une critique du monde tel qu'il va et des «grandes personnes » qui le font ce qu'il est. Il met en cause leur mode même d'appréhension et de compréhension du monde, il n'hésite jamais à questionner les idées reçues de ces "grandes personnes » qui "se voient importantes comme des baobabs». Qu'est-ce donc en effet qui caractérise les « grandes personnes"? Elles "sont décidément très très bizarres" et le plus bizarre est leur incapacité fondamentale à discerner ce qui est important à l'exemple de ce "monsieur cramoisi » du chapitre VII :

Il n'a jamais respiré une fleur. Il n'a jamais regardé une étoile. Il n'a jamais aimé personne. Il n'a jamais rien fait d'autre que des additions. Et toute la journée il répète comme toi : «Je suis un homme sérieux ! Je suis un homme sérieux ! » et ça le fait gonfler d'orgueil ».

12 Cette idée est omniprésente, presque chaque personnage le démontre, sur chacune des planètes visitées par le Petit Prince. On pense évidemment au vaniteux (chapitre XI) qui veut seulement qu'on l'admire, mais que dire du roi (chapitre X) qui cherche des sujets? Et du géographe qui ne veut écrire que des choses "éternelles» ou du businessman (chapitre XIII) qui se dit "sérieux» parce qu'il compte et recompte, "c'est difficile, mais je suis un homme sérieux!»? Compter: c'est bien aussi une caractéristique de la grande personne, elle aime les chiffres, se cache derrière eux et les calculs, les additions tout particulièrement. "Les grandes personnes aiment les chiffres ", parce qu'ils donnent l'illusion du sérieux, du savoir et de la possession, alors que «nous qui comprenons la vie, nous nous moquons bien des numéros ». Mesurer et compter ne suffit pas à connaître et comprendre, mais c'est utile pour convaincre les "grandes personnes": "si je vous ai raconté ces détails sur l'astéroïde B 612 et si je vous ai confié son numéro, c'est à cause des grandes personnes ». Sans en avoir l'air, le Petit Prince nous rappelle aussi que compter et mesurer est acte de pouvoir. Or " rien de ce qui concerne l'homme ne se compte, ni ne se mesure " (Pilote de guerre : 160). Chiffres et argent sont liés, et aussi l'incapacité à dépasser les apparences. L'épisode de l'astronome turc (chapitre IV) l'indique très tôt dans l'ouvrage : lors d'une première communication scientifique à un congrès d'astronomie en 1909 sur l'astéroide B 612 (qu'il a découverte), il n'est écouté par personne; une seconde présentation, en 1920, sur le même sujet, lui assure la reconnaissance scientifique. C'est qu'entre temps il a changé de 
costume... parce qu'un « dictateur turc imposa à son peuple, sous peine de mort, de s'habiller à l'européenne ».

Comme elles ne savent pas voir ce qui est important, les grandes personnes sont aussi tristes. Elles se distraient de cette tristesse en comptant, ou bien elles tombent dans des cercles vicieux, "s'agitent et tournent en rond", comme le buveur (chapitre XII) sur sa planète, qui boit pour oublier sa honte de boire, comme les voyageurs de la planète du chapitre XXII qui voyagent sans savoir où ils vont, seulement parce que « on est jamais content là où l'on est ". Ils ne savent pas ce qu'ils cherchent : "seuls les enfants savent ce qu'ils cherchent ». Le Petit Prince lui-même a bien failli tomber dans le piège : il est parti de sa planète sans bien savoir ce qu'il cherchait, il est parti pour fuir parce qu'il avait «des difficultés avec une rose ». Or partir, bouger, ne sert à rien qu'à faire regretter d'être parti. Le paradoxe est que si le mouvement est bien un déracinement il est aussi la condition pour connaître la valeur de l'enracinement, de l'attachement au lieu que l'on quitte. En d'autres termes, ce que les " grandes personnes » ont perdu est leur capacité à faire lieu, et leur mouvement devient quête du lieu, quête qui elle même pousse au mouvement qui lui-même empêche de faire lieu. C'est ce que le Petit Prince va comprendre, accédant alors à une nouvelle compréhension de son monde. C'est le renard (chapitre XXI) qui, sur la terre (qui est la septième planète visitée), va lui faire don de la clé : « on ne voit bien qu'avec le cour. L'essentiel est invisible pour les yeux». Mais pour bien voir il faut prendre le temps, et même le perdre : le temps d'apprivoiser, le temps d'aimer. Sur trois planètes visitées au moins le Petit Prince rencontre des personnages obsédés par le temps. Le businessman le lui dit clairement: "je n'ai pas le temps de flâner ». Le marchand de pilule (chapitre XXIII) quant à lui vent des pilules qui coupent la soif et font gagner le temps que l'on passe à boire (" on épargne cinquante-trois minutes par semaine » précise le marchand, « les experts ont fait des calculs»). L'allumeur de réverbère est au contraire une victime d'un temps qui s'accélère. Sa planète (chapitre XIV) est la plus petite de celles visitées par le Petit Prince, l'allumeur y vit seul et son rôle est d'allumer le réverbère au coucher du soleil et de l'éteindre à son lever. Comme sa planète tourne de plus en plus vite, il doit allumer et éteindre de plus en plus souvent, presque jusqu'au clignotement :

- C'était raisonnable autrefois. J'éteignais le matin et j'allumais le soir. (...)

- Et, depuis cette époque, la consigne a changé ?

- La consigne n'a pas changé, dit l'allumeur. C'est bien là le drame! La planète

d'année en année a tourné de plus en plus vite, et la consigne n'a pas changé ». 
Figure 1. L'allumeur de réverbère

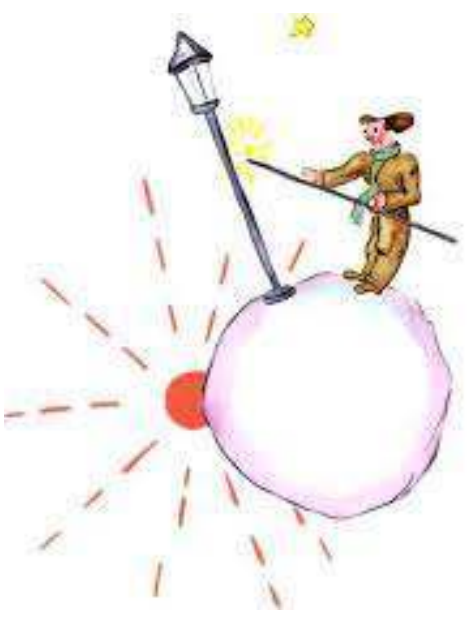

Source : Antoine de Saint-Exupéry, Le Petit Prince, 1943. comme la plus dangereuse des dérives. Il est question ici de la compression de l'espacetemps, très précisément ce que décrypte David Harvey (1990) : une planète dont la taille se réduit par la réduction de la distance-temps. L'urgence devient alors de ralentir, de faire l'éloge de la lenteur à la manière de Pierre Sansot (1998), ou bien de refuser d'allumer le réverbère, c'est-à-dire de faire changer la «consigne » d'un monde qui fait, comme l'explique le renard au Petit Prince, que «les hommes n'ont plus le temps de rien connaitre $~_{4}$. Ce serait une révolte, sans doute même une révolution: s'arrêter c'est protester, s'arrêter, ralentir, devient un acte politique qui ré-introduit le temps dans l'espace. Ce qui n'est pas une proposition nouvelle puisqu'Henri Lefebvre la faisait déjà : ressusciter le temps qui est « tué par la société » (Lefebvre, 1974 : 115), ou plus précisément le ré-introduire dans l'espace puisque c'est résister au "primat de l'économique et plus encore celui du politique (qui) entraîne la suprématie de l'espace sur le temps. (...) Cette évacuation apparente du temps ne serait-elle pas un des traits caractéristiques de la modernité? » (114). Mais comment ralentir et retrouver le temps dans l'espace, interroge Saint-Exupéry, alors même que nous vivons à la fois un écartèlement permanent, comme le Petit Prince mais aussi comme l'aviateur, entre le désir d'ancrage en un lieu et désir de mouvement et sous la pression d'une société dont le fonctionnement même repose sur une nécessaire compression du temps? C'est aussi une réflexion très contemporaine et qui concerne directement la géographie en tant que science sociale. Et l'aviateur ici est contraint de s'arrêter : il est tombé en panne dans le désert, il ne l'a pas voulu mais c'est la condition nécessaire pour que, dans le désert, le monde lui devienne lieu.

Alors quoi ? Si ce n'est pas compter, si ce n'est pas poursuivre le temps qui fuit, qu'est-ce qui compte? Peut-être ce qui semble ne pas compter, ce qui est perdu. En effet, ce que l'on ne peut pas compter, ni mesurer, ni peser, est unique. Mais n'est unique que ce que l'on construit en soi-même comme unique à force de temps perdu. Le Petit Prince court le risque d'être trompé: "Je me croyais riche d'une fleur unique, et je ne possède qu'une rose ordinaire». Justement non... il va lui falloir apprendre que «c'est le temps que tu as perdu pour ta rose qui fait ta rose si importante ». 


\section{L'avion et la maison, le mouvement et l'ancrage}

Dès lors, nous nous sentîmes perdus dans l'espace interplanétaire, parmi cent planètes inaccessibles, à la recherche de la seule véritable, de la nôtre, de celle qui, seule, contenait nos paysages familiers, nos maisons amies, nos tendresses. (Terre des hommes, p. 182)

Une double quête, de l'envol et du mouvement d'une part, de la maison et de la tendresse d'autre part, caractériserait pour Saint-Exupéry la condition humaine et serait l'obstacle majeur qui empêche de "faire lieu ». Tuan voit dans cette même dualité l'essence même de ce qui fonde notre relation à l'espace : « place is security, space is freedom. We are attached to one and long for the other» (1971: 3). Saint-Exupéry illustre l'impossibilité de conjuguer les désirs de liberté et de sécurité par l'exemple des gazelles qu'il élevait quand il était en poste à l'escale de Juby, fortin espagnol isolé sur la côte de l'Atlantique entre Casablanca et Dakar. Tant qu'elles sont jeunes, on peut croire ces gazelles apprivoisées, elles viennent vous manger dans la main, on les caresse :

Mais vient le jour où vous les retrouvez, pesant de leurs petites cornes, contre l'enclos, dans la direction du désert. (...) Ce qu'elles cherchent (...) c'est l'étendue qui les accomplira. (...) Elles veulent connaître la fuite rectiligne, coupée de brusques jaillissements (...) qu'importe les chacals (...) les voilà prises de nostalgie. La nostalgie c'est le désir d'on ne sait quoi... Il existe, l'objet du désir, mais il n'est pas de mots pour le dire (Terre des hommes, p. 275).

On sait la diversité des formes de nostalgie (Gervais-Lambony, 2012), et surtout la dualité de la notion (Boym, 2000) qui peut signifier désir de retour vers le passé ou au contraire permettre de se tourner vers l'avenir avec la conscience du caractère éphémère du présent. La nostalgie dont parle ici Saint-Exupéry est bien tournée vers le futur, elle est désir d'un accomplissement à travers le mouvement. C'est une des raisons du départ du Petit Prince de son astéroïde, départ qui va lui faire connaître ensuite l'autre versant de la nostalgie, celle de ce qu'il a quitté, qui va le mener au désir de retour. Mais le retour nostalgique n'est certainement pas retour vers l'identique : "la nostalgie s'écrit au futur antérieur, et tel est sans doute le temps de toutes les fondations, qui ne sont que des refondations (... ): il ne s'agit pas de reproduire à l'identique, mais de fabriquer de l'autre » (Cassin, 2013:67). Cette nostalgie qu'il apprend à vivre a donc fait prendre conscience au Petit Prince de l'illusion dont il a été victime, comme les gazelles, selon laquelle on ne serait libre que dans le mouvement et que l'on ne s'accomplit qu'en partant de chez soi (et en sortant de soi) vers « l'étendue » au sens matériel.

Cette notion d'étendue, concept géographique par excellence, est approfondie par SaintExupéry dans Pilote de guerre, texte contemporain de l'écriture du Petit Prince. Le personnage et narrateur de Pilote de Guerre survole, en 1939, le nord de la France en guerre lors d'une mission aérienne de renseignement qui doit le mener par-delà les lignes allemandes jusqu'à Arras. Il fait, en plein ciel, une expérience qui l'étonne : " je découvre de mes dix mille mètres un territoire de l'envergure d'une province, et cependant tout s'est rétréci jusqu'à m'étouffer. Je dispose ici de moins d'espace que je n'en disposais dans ce grain noir " ( : 159). Ce " grain noir » est la manière dont apparait à l'œil du pilote une maison, et c'est la maison de son enfance que cette vision lui évoque. Et dans son souvenir, cette maison, une seule pièce même de cette maison, est un espace bien plus vaste que les terres qu'il survole : après celui d'étendue c'est donc le concept d'échelle que Saint-Exupéry met en cause. L'étendue n'est pas une question de dimension, de superficie, elle ne se mesure 
pas : «l'étendue véritable n'est point pour l'œil, elle n'est accordée qu'à l'esprit. (...) Une mauvaise littérature nous a parlé du besoin d'évasion. Bien sûr, on s'enfuit en voyage à la recherche de l'étendue. Mais l'étendue ne se trouve pas. Elle se fonde » (:160). Echelle et étendue relèvent donc des représentations et du sentiment, elles sont liées à l'ordonnancement que seule la relation à l'autre rend possible: "Quand un hasard éveille l'amour, tout s'ordonne dans l'homme selon cet amour, et l'amour lui apporte le sentiment de l'étendue. (...) Le pathétique, c'est le sentiment de l'étendue » (:161).

19 Les gazelles sont donc dans l'illusion, le Petit Prince aussi quand il prend son envol, et Saint-Exupéry lui-même bien sûr aussi. La contradiction entre mobilité et ancrage est une illusion. La vraie question est : « que valons-nous une fois immobiles? (Pilote de guerre : 160), comment accéder à l'étendue dans l'immobilité ? Saint-Exupéry en donne trois exemples : le religieux qui prie, le savant qui travaille, l'artiste qui peint. Ce dernier c'est ici Cezanne devant sa toile : «il n'est jamais plus homme que lorsqu'il se tait, éprouve et juge. Alors sa toile lui devient plus vaste que la mer » (: 160). Tout à fait inutile donc, pour le dire en termes familiers, « d'accélérer le mouvement »... pour aller plus vite et plus loin : l'étendue est à l'intérieur de nous, elle nous vient des lieux du passé.

20 La planète du Petit Prince est toute petite, et il ne prend conscience de son importance pour lui qu'au moment où il la quitte. A la fin, c'est le désir de retour qui l'emportera: malgré sa peur du serpent et sa peur de la mort, le Petit Prince retourne chez lui, il y sera re-fondateur. L'adjectif que le narrateur-aviateur utilise pour décrire le sentiment du Petit Prince est « mélancolique » (chapitre VI). Cette mélancolie est regret : regret d'être parti, regret d'avoir quitté sa maison, son lieu, sa planète. Il l'a quitté sur un malentendu : sa fleur (sa rose) se plaint sans cesse, n'est jamais satisfaite, ne lui déclare son amour que le jour où il s'en va. Sur sa planète, pourtant, il faisait tout de son mieux, s'occupait de ramoner ses deux volcans, arrachait les mauvaises herbes, luttait contre les baobabs qui auraient pu, si on les avait laissés pousser, envahir tout l'espace, et surtout il faisait tout pour protéger sa rose jusqu'à la mettre sous globe pour lui éviter les courants d'air: "Tous ces travaux familiers lui parurent, ce matin-là, extrêmement doux. Quand il arrosa une dernière fois la fleur, et se prépara à la mettre à l'abri sous son globe, il se découvrit l'envie de pleurer » (chapitre VIII). Mais il se sentait seul et ne se savait pas aimé. Alors il s'est envolé aussi par désir : il était déjà mélancolique sur sa planète ou il n'avait « pour distraction que la douceur des couchers de soleil » (chapitre VI). Rester ou partir, s'ancrer ou bouger, il faudrait les deux à la fois; mais, chez Saint-Exupéry, le mouvement est la condition de l'ancrage (on ne bouge que parce que l'on cherche à faire lieu) celui-ci n'est rendu possible que par la nostalgie.

21 Le but du voyage du Petit Prince devient alors de mieux comprendre ce qui l'attache à sa planète et donc à son passé, il s'agit d'apprendre à devenir nostalgique. Le texte devient métaphore du parcours de la vie : quitter son enfance pour la comprendre, la comprendre pour vouloir y retourner, n'avoir d'autre choix que de mourir pour y parvenir. Et entretemps apprendre à aimer, apprendre ce qui fait qu'une relation est unique. Une fois arrivé sur la terre, grâce aux conseils du géographe, le Petit Prince va connaître plusieurs chocs. Le premier est sa confrontation avec un jardin de roses, toutes pareilles...

Le Petit Prince réalise, à ce moment-là, l'exercice comparatif par excellence : grâce à la comparaison il parvient à dégager le singulier de l'universel. C'est en comparant qu'il apprend que derrière ressemblances et similitudes se cache le caractère unique des choses et des lieux. En d'autres termes il découvre que «l'unicité du Monde et de chacun de ses lieux constitue un cadre conceptuel majeur de toute analyse des ressemblances entre les objets 
géographiques » (Levy, 1999 :133). C'est le renard qui lui explique cela : comment devenir unique, comprendre ce qui vous attache à un lieu. Le renard appelle cela "apprivoiser ». Que dirions nous? « Habiter» (Berque, 1996; Stock, 2004 ; Lazzarotti, 2006) à mon avis, habiter au sens de rendre unique ce qui nous environne, y créer des liens avec objets et paysages pour que chaque chose finisse par être évocatrice de sentiments pour d'autres êtres. Ainsi le renard et le blé : «le blé est pour moi inutile. Les champs de blé ne me rappellent rien. (...) Mais (...) quand tu m'auras apprivoisé! Le blé, qui est doré, me fera souvenir de toi. Et j'aimerai le bruit du vent dans le blé... ». C'est ainsi que se constitue une relation unique avec le lieu, faite de mise en relation affective et de construction d'une mémoire de ce lieu : il évoque mais il ne peut évoquer que ce qui est absent, ce que l'on a perdu. Tout dans le lieu devient alors unique, ce qu'Eric Dardel (1952) a appelé « l'intimité avec la Terre » est rétabli. Cette intimité est ce qui ne peut pas se compter ni s'acheter comme le Petit Prince l'explique au businessman: ce qui est unique n'est pas reproductible donc ne peut devenir marchandise (Lefebvre, 1974). Certes, il s'agit aussi d'apprendre à aimer l'absence : «-Mais tu vas pleurer (...) alors tu n'y gagnes rien! -J'y gagne, à cause de la couleur $d u$ blé». C'est le risque qu'il y a à apprivoiser et à aimer, mais c'est aussi la condition nécessaire pour « faire lieu ».

23 Est-ce cela la manière d'« habiter en poète » (Heidegger, 1958) ? Est-ce cela rendre un lieu habitable car peuplé de ce que Michel de Certeau (1980) appelle des « revenants » ? Cela ressemble fort, en tous cas, à une définition phénoménologique de la production du lieu : projection de soi hors de soi, dans les choses et les lieux, ceux-ci n'existant qu'à cette condition (Berque, 1996; Hoyaux, 2013). C'est ce qu'explique le Petit Prince aux roses de la terre : «vous êtes belles, mais vous êtes vides, (...) personne ne vous a apprivoisées et vous n'avez apprivoisé personne " (chapitre XXI). La relation aux choses et aux lieux, celle du Petit Prince à sa planète et surtout à sa rose, est une relation amoureuse : c'est l'amour qui apporte l'étendue, c'est l'amour qui ordonne le monde pour chacun. La manière dont Saint-Exupéry décrit la relation au lieu est très précisément conforme aux figures du discours amoureux analysées pas Roland Barthes (1977): le ravissement (ou coup de foudre du premier contact, à l'éclosion de la rose), le vouloir-saisir (l'amoureux cherche à comprendre l'autre et reste toujours sur sa faim, il interroge la rose), le désir de s'abîmer (de disparaître en l'autre, de se fondre), la compassion (pour le sujet aimé quand on le voit dans la peine quand la rose se plaint des courants d'air), le désir d'union (jamais assouvi, impossible, et qui provoque la rupture, ici le départ vers d'autres planètes). Dans l'ensemble, on comprend qu'il est question d'une relation à l'Autre, à la fois souffrance et bonheur de cette altérité, à la fois aussi physique et psychologique. Mais cet autre est en même temps soi-même et en même temps a besoin du lieu. Plus que le mot lieu c'est ici le mot maison qui convient, chez Saint-Exupéry la figure de la maison est récurrente et presque toujours posée tout à la fois en opposition et en lien avec le ciel et le désert. Ce dernier tout particulièrement est l'espace ouvert de l'errance et du voyage. Il attire et donne la liberté. Le pilote le voit d'en haut, s'en émerveille, de même qu'il s'émerveille du ciel étoilé qu'il traverse. Mais dans le désert point de maison, point d'ancrage. Et c'est pourtant dans le désert qu'il faut aller pour savoir ce qu'est la maison car c'est là qu'elle se révèle à l'esprit et qu'on la découvre capable de "remplir ma nuit de sa présence » (Terre des Hommes : 207). Car :

le merveilleux d'une maison n'est point qu'elle vous abrite ou vous réchauffe, ni qu'on en possède les murs. Mais bien qu'elle ait lentement déposé en nous ces provisions de douceur. Qu'elle forme, dans le fond du cœur, ce massif obscur dont 
naissent, comme des eaux de source, les songes... Mon Sahara, mon Sahara, te voilà tout entier enchanté par une fileuse de laine! (Terre des hommes : 209).

Il n'y a donc antinomie absolue ni entre la maison et le désert, ni entre l'ancrage et le mouvement, ni entre le passé et le présent. Au contraire, dans chacune de ces apparentes oppositions binaires, l'un des termes est condition de l'autre et réciproquement: c'est dans le mouvement que l'on comprend son ancrage, c'est dans l'ancrage que l'on rêve de mouvement ${ }^{5}$; le présent est envahi par le passé qui lui même n'existe que dans le présent. Et tout comme le Petit Prince emporte le souvenir sans cesse plus vivace de sa planète et de sa rose, on emporte dans l'avion celui de sa maison, dans le mouvement celui de son lieu. La maison du Petit Prince aurait d'ailleurs pu être en ce sens idéale : astéroïde plutôt que planète, elle est en mouvement, réalisant ainsi la synthèse parfaite entre maison et avion, entre mouvement et ancrage. Maison et désert sont deux modalités complémentaires de notre spatialité, également désirables ou plus précisément désirables alternativement et nécessaires l'un à l'autre.

Saint-Exupéry a commencé cette réflexion sur cette tension entre mouvement et ancrage très tôt puisqu'elle est déjà le sujet de Courrier Sud, son premier roman, publié en 1929: l'aviateur Bernis revient à Paris, après deux ans passés dans le Sahara, vers son amour de jeunesse, Geneviève. Elle représente son passé et son enracinement, il représente le mouvement, l'appel au départ. Il tente de l'arracher au passé et aux lieux du passé, elle ne peut pas le supporter. Elle mourra, il repartira, et son vol de Toulouse à Dakar pour l'Aéropostale sera une nouvelle fuite mais cette fois sans retour possible. Il faut donc à la fois l'avion qui vous emmène et la maison qui vous fixe mais qui devient inaccessible, si l'on y revient ce ne peut être que comme un fantôme de ce que l'on fut. C'est pourquoi le Petit Prince doit mourir. Et comprenons bien que le temps et l'espace, à ce jeu de la vie, ne font qu'un: l'attachement au lieu est fait du souvenir des êtres chers (chers car apprivoisés), il est donc fait du temps passé.

Aimer sa rose, pour le Petit Prince, est donc désir de fusion, mais c'est impossible, bien sûr :

les liens entre un être et nous n'existent que dans notre pensée. (...) malgré l'illusion dont nous voudrions être dupes et dont, par amour, par amitié, par politesse, par respect humain, par devoir, nous dupons les autres, nous existons seuls. L'homme est l'être qui ne peut sortir de soi, qui ne connaît les autres qu'en soi, et, en disant le contraire, ment (Marcel Proust, Albertine disparue : 34 ).

On fait donc exister des lieux en nous-mêmes, ou plus précisément on utilise des lieux pour nous faire exister nous-mêmes, on les quitte puis on y revient, on tente de se quitter soi même puis on s'efforce d'y revenir, au vrai soi, qui est temps passé et lieu perdu. Chez Proust la solution existe, elle est donnée par la mémoire involontaire qui permet de remonter le temps et l'espace, de revivre l'expérience passée. Chez le Saint-Exupéry du Petit Prince seule la mort permet le retour vers le temps et le lieu de l'enfance. La touche d'espoir est peut-être dans la conclusion du Petit Prince : le dessin d'un paysage saharien, vide à l'exception d'une étoile dans le ciel. «Le plus beau et le plus triste paysage du monde (... ) c'est ici que le petit prince a apparu sur terre, puis disparu » : la disparition est devenue trace, la beauté est faite de cette tristesse, une rencontre humaine a fait d'un lieu désert un lieu habitable car habité par le souvenir. Le désert est désormais habitable.

8 Il faut donc s'endormir. Car comme le dit le Petit Prince à l'aviateur: "j'aurai l'air d'être mort et ce ne sera pas vrai... » (chapitre XXVI). Saint-Exupéry nous livre des clés pour comprendre le Petit Prince dans Terres des hommes. Dormir c'est rêver, rêver c'est à la fois 
le moyen de remonter le temps et l'espace, involontairement, c'est aussi « un pouvoir admirable : celui de refuser le monde présent » ( : 272). Et c'est précisément dans le désert que Saint-Exupéry dit en avoir pris conscience. En 1935, alors qu'il tente de battre le record de temps pour joindre Toulouse à Saigon, son avion s'écrase dans le désert libyen. Lui et son mécanicien survivent trois jours durant, errants, sauvés finalement par deux bédouins arabes. Cet incident est souvent évoqué comme une des sources d'inspiration pour Le Petit Prince : celui-ci est tout aussi réel que les mirages décrits dans Terre des hommes. Mirages et rêves se rencontrent ici, dont il faut se réveiller, réveil que Saint-Exupéry définit comme la "reprise en charge de la vie, ce rêve que l'on ne choisit pas » (: 273).

Disons alors que le Petit Prince n'est pas mort, il dort. Et il laisse sa trace puisqu'il est parvenu à ré-enchanter le monde. C'est-à-dire ? Le restituer en ce qu'il a d'unique, en ce qu'il est notre lieu et donc une " œuvre »: "s'il en est ainsi, tout espoir n'est pas perdu de retrouver un mouvement dialectique tel que l'œuvre traverse le produit et que le produit n'engloutisse pas la création dans le répétitif» (Lefebvre, 1974 : 93). On peut être surpris que je donne ici le mot de conclusion à Henri Lefebvre. Il ne faut pourtant pas s'y tromper : d'une part de nombreux passages de l'œuvre de Lefebvre rejoignent les inquiétudes de Saint-Exupéry sur la destruction de la relation aux lieux sous les coups du capitalisme; d'autre part parler de la relation humaine au lieu c'est très directement parler d'un droit, droit des humains sur la Terre des hommes, droit de faire lieu, donc œuvre.

\section{BIBLIOGRAPHIE}

BARTHES R. (1977), Fragments d'un discours amoureux, Paris, Editions du Seuil, 242p.

BERNANOS G. [1946] (2015), La France contre les Robots, Paris, Le Castor Astral, 330p.

BERQUE A. (1996), Etre humains sur la terre, Paris, Gallimard, 212p.

BOYM S. (2001), The Future of Nostalgia, New York, Basic Books, 403p.

CAMUS A. (1953), L'exil d'Hélène, in L'été, Paris, Gallimard, pp. 133-140.

CASSIN B. (2013), La nostalgie. Quand donc est-on chez soi ?, Paris, Autrement, 149p.

COLLOT M. (2014), Pour une géographie littéraire, Paris, Editions Corti, 271p.

DARDEL E. [1952] (1990), L'Homme et la Terre. Nature de la réalité géographique, Paris, CTHS, 199p.

DE CERTEAU M., GIARD L., MAYOL P. (1980), L'invention du quotidien, Vol 2. Habiter, cuisiner, Paris, Gallimard, 416p.

GERVAIS-LAMBONY P. (2012) « Nostalgies citadines en Afrique Sud », Espaces/Temps.net http://www.espacestemps.net/articles/nostalgies-citadines-en-afrique-sud/ consulté le 30 juillet 2015.

HARVEY D. (1990), The condition of Postmodernity, New York, Blackwell, 378p.

HEIDEGGER M. (1958), Essais et conférences, Paris, Gallimard, 232p. 
HOYAUX A.-F. (2013), « Géographie et phénoménologie : perspectives théoriques et méthodologiques autour de la proximité et de l'authenticité », in FRERE B., LAOUREUX S. (dir.), La phénoménologie à l'épreuve des sciences humaines, Berne, Peter Lang, pp. 73-88.

LAZZAROTTI O. (2006), Habiter, la condition géographique, Paris, Belin, 228p.

LEFEBVRE H. (1974), La production de l'espace, Paris, Anthropos, 486p.

LEVY J. (1999), Le tournant géographique. Penser l'espace pour lire le monde, Paris, Belin, 400p.

PAQUOT T. (2005), Demeure terrestre. Enquête vagabonde sur l'habiter, Paris, Editions de l'Imprimeur, 71p.

PROUST M. (édition de 1987), La Recherche du temps perdu, 5 volumes, Paris, Gallimard.

SAINT-EXUPERY A. (édition de 1994), CEuvres complètes, 2 volumes, Paris, Gallimard.

SANSOT P. (1998), Du bon usage de la lenteur, Paris, Payot, 204p.

STOCK M. (2004), L’habiter comme pratique des lieux géographiques, EspacesTemps.net.

TUAN Y-F. (1971), Space and Place. The perspective of experience, Minneapolis, University of Minnesota Press, 235p.

\section{NOTES}

1. Né avec le XXème siècle, d'une famille aristocratique, Antoine de Saint-Exupéry devient pilote lors de son service militaire en 1921. En 1926 il est engagé par la compagnie Latécoère (future Aéropostale intégrée ensuite à Air France) qui depuis Toulouse ouvre une ligne aérienne de courrier postal d'abord jusqu'à Dakar puis vers l'Amérique du Sud, Argentine d'abord Chili ensuite en 1929. Après la faillite de l'Aéropostale en 1932, il devient journaliste-aviateur indépendant. En 1939 il est mobilisé et est pilote pendant la seconde guerre mondiale. Exilé aux Etats-Unis après la capitulation de la France, il obtient d'être remobilisé, malgré son âge et son état de santé, en 1944 pour participer aux opérations du débarquement en Provence (c'est de l'une de ces missions de reconnaissance aérienne qu'il ne revint pas). Parallèlement à cette vie de pilote, il écrit et publie, obtenant succès et reconnaissance internationale d'écrivain (Prix Fémina pour Vol de nuit en 1931, Grand prix du roman de l'Académie française pour Terre des Hommes en 1939).

2. Toutes les citations des textes de Saint-Exupéry sont tirées de l'édition de la collection «Bibliothèque de la Pléiade», Paris, Gallimard, 1994. Pour celles du Petit Prince on indique le chapitre, pour celles de Terre des hommes et de Pilote de guerre la pagination.

3. Léon Werth est un ami de Saint Exupéry, écrivain proche du parti communiste. Issu d'une famille juive, il a choisi de rester en France occupée et de rejoindre la résistance (La Lettre à otage, publiée en 1942, lui est aussi adressée).

4. Il serait possible aussi de lire les évolutions du monde de la recherche à la lumière du réverbère: un monde où le temps se réduit sans cesse sous les effets conjugués des réformes successives et des temporalités toujours raccourcies de la recherche contractualisée. On peut penser d'ailleurs que prendre le temps d'écrire un article sur le Petit Prince est une manière de réaction à cette compression du temps.

5. Dans ces travaux sur l'habiter et la mobilité (notamment le tourisme comme forme de mobilité) Mathis Stock (2004) est amené à se poser une question du même ordre : comment conjuguer habiter et mobilité ? La mobilité selon lui induit un nouveau mode d'habiter, ce qui est une manière de réponse à Saint-Exupéry. 


\section{RÉSUMÉS}

En s'appuyant sur l'œuvre d'Antoine de Saint-Exupéry ce texte propose une réflexion sur la condition humaine et la production des lieux. Ceux-ci sont définis comme des espaces rendus uniques par les relations humaines qui s'y nouent puis qu'ils évoquent pour leurs habitants. Analyser les lieux suppose à la fois de comprendre la place du temps (et de son accélération) dans leur production et comment peut être surmontée la tension entre désir de mouvement et désir d'ancrage. Le Petit Prince, et plus largement l'œuvre entière de Saint-Exupéry, sont marqués par cette tension et la difficulté qu'il y a à la surmonter. La surmonter signifierait idéalement réussir un "voyage immobile» dans l'espace et dans le temps. Cela passe par une réflexion sur la nostalgie, sur l'enfance et le passé.

In this paper the author analyses the relation between time an place through a study of the work of Antoine de Saint-Exupéry. Place is defined as a space made unique by relations between humans that it then evoques. This form of production of place relies on the one hand on a timespace relation, of the other hand on the capacity to overcome the tension between contradictory desires: desire for movement, desire for home. The Little Prince, and all the writings of SaintExupéry are an effort to find a solution to this contradiction. Solution might be immobility in a changing space and a poassing time, it has to be seeked in a specific relation to the past and childhood but also in a certain definition of nostalgia.

INDEX

Thèmes : Carnets de recherches

\section{AUTEUR}

\section{PHILIPPE GERVAIS-LAMBONY}

